\title{
Les Lettres nouvelles de Milan de Pierre Gringore : la propagande entre vers et prose ${ }^{1}$
}

Le 10 avril 1500, le duc de Milan Ludovic Sforza (le More) est capturé et livré à Louis de La Trémoille, lieutenant-général de Louis XII en Milanais. Alors que la ville était aux mains des Français depuis juillet 1499, Ludovic était revenu au début de l'année 1500, à la faveur d'un soulèvement des Milanais contre les Français. Sa capture permet donc d'écarter toute nouvelle menace sur Milan; elle couronne aussi les velléités de Louis XII sur un duché qu'il estime lui revenir de droit par sa grand-mère, Valentine Visconti. L'événement doit donc être relayé et célébré à sa juste mesure au royaume de France. Pierre Gringore est de ceux qui s'attellent à cette tâche. Alors qu'il n'est encore que l'auteur d'une œuvre morale, le Chasteau de Labour, parue un an plus tôt ${ }^{2}$, il compose à l'occasion de la capture du More les Lettres nouvelles de Milan. Avec les regretz du seigneur Ludovic ${ }^{3}$, œuvre imprimée sous la forme d'une plaquette de six feuillets, en 1500, chez Pierre Le Caron. Le texte se compose tout d'abord de trois lettres qui racontent respectivement les circonstances de la victoire française à Milan, la capture du duc et la liesse des Parisiens lorsqu'ils apprennent ces nouvelles : elles sont rédigées en prose par le roi pour la première, «Loys » et «Robertet » pour la suivante ${ }^{4}$, tandis que la troisième, écrite depuis Paris et non Milan, demeure anonyme. Elles sont suivies d'un débat, en vers, opposant Français et Italiens : mais le débat n'est que de façade, car les deux partis s'accordent pour reconnaître la supériorité militaire et morale de Louis XII et de son peuple. Suivent également en vers des «regretz» formulés par le More captif, qui rappellent encore les événements déjà détaillés dans les lettres et les vers précédents. L' « acteur » conclut enfin par un huitain. Quel est le sens d'un tel dispositif ${ }^{5}$ dans le cadre d'une écriture de propagande et, en particulier, pourquoi revenir plusieurs fois au récit des mêmes événements sous différentes formes ou dispositifs énonciatifs ? Notre hypothèse est que ces versions endossent chacune des objectifs rhétoriques différents, entre information et célébration, et s'adressent à des publics variés, parfois opposés, alors que le jeune auteur s'efforce sans doute à la fois de toucher un lectorat urbain, avide de nouvelles du front, tout en se faisant remarquer par le pouvoir, dans l'espoir de trouver un éminent mécène. Après avoir éclairé les enjeux des Lettres nouvelles de Milan dans la carrière de Gringore, nous analyserons dans ce texte l'articulation des vers à la prose, ainsi que les aspects et valeurs, parfois contradictoires, que revêt l'écriture poétique et ses différents styles dans la perspective d'une propagande de l'action militaire de Louis XII outremonts.

\footnotetext{
${ }^{1}$ Cet article est le développement d'une présentation réalisée à la $64^{\mathrm{e}}$ conférence annuelle de la Renaissance Society of America (Nouvelle-Orléans, 22-24 mars 2018), lors d'une table-ronde organisée par Olga Anna Duhl et Nathalie Dauvois, intitulée «Gringore, écrivain, homme de théâtre et son rapport au pouvoir ».

${ }^{2}$ Sur la carrière de Gringore, voir Charles Oulmont, Pierre Gringore : la poésie morale, politique et dramatique à la veille de la Renaissance, Genève, Slatkine Reprints, 1976 [Paris, 1911].

${ }^{3}$ Le texte a fait l'objet de deux éditions modernes : l'une par Enea Balmas (Milano-Varese, Cisalpino, 1955) et l'autre par Cynthia Brown dans Gringore, Euvres polémiques rédigées sous le règne de Louis XII, Genève, Droz, 2003, p. 63-95. Toutes nos références aux Lettres nouvelles de Milan proviennent de cette seconde édition.

${ }^{4}$ Dans son édition des Chroniques de Louis XII de Jean D'Auton, René de Maulde La Clavière explique que sitôt que le roi fut informé de la capture de Ludovic, il fit imprimer ses lettres afin de répandre la nouvelle dans son royaume (Paris, Renouard, 1889, vol. I, n. 3, p. 266-367).

${ }^{5}$ Cette question a déjà été évoquée par Cynthia Brown dans The Shaping of History and Poetry in Late Medieval France, Birmingham, Alabama, Summa Publications, 1985, p. 38-43. Elle souligne déjà l'opposition entre une prose véridique, où nul narrateur n'intervient pour commenter les faits, et des vers qui ne sont que commentaires politiques : nous chercherons à approfondir ses remarques.
} 


\section{Propagande $^{6}$ et protection}

Par cette publication de circonstance, Gringore semble avoir activement recherché une protection royale, ou du moins un mécène éminent. Cette entreprise ne s'acheva en réalité qu'avec son installation définitive à la cour de Lorraine, à partir de 1518 ; avant, différentes dédicaces placées en tête de ses poèmes, adressées à de puissants seigneurs ou prélats comme Jacques d'Estouteville ou l'évêque de Cahors ${ }^{7}$, soutiennent l'idée que Gringore cherche la faveur des grands. Faute d'appartenir à des cercles courtisans, il se tourne, dès les premières années du XVI ${ }^{\mathrm{e}}$ siècle, vers l'imprimerie afin de diffuser largement son œuvre. Même si cela ne permet pas de tirer des revenus conséquents (car la protection d'un mécène demeure la source de rémunération la plus importante et la plus stable que peut rechercher un auteur qui n'aurait pas d'autres charges lui procurant des revenus ${ }^{8}$ ), Gringore peut ainsi toucher un vaste public. Celui-ci peut, en retour, doublement lui attirer les faveurs d'un protecteur éminent, parce qu'il aura remarqué son talent d'écrivain et parce qu'il aura constaté son aptitude à défendre spontanément ${ }^{9}$ et efficacement la politique royale auprès d'une large audience urbaine. Il ne s'agit pas pour autant de manipuler ou même constituer une opinion publique, mais d'expliciter les principes, souvent historiques et moraux, guidant l'action politique royale $^{10}$. Cette stratégie s'avéra efficace pour Jean Lemaire de Belges, qui rejoignit la cour de France à partir de 1512, après avoir publié Le traictie intitule de la difference des schismes et des concilles de leglise ${ }^{11}$, dans lequel il défend la politique de Louis XII contre le pape Jules II. Mais Gringore ne connaîtra pas un tel succès avec la cour royale ${ }^{12}:$ il faut dire que

\footnotetext{
${ }^{6}$ Nous entendons ce terme non comme une campagne de communication concertée, provenant de la couronne, pour soutenir ses desseins diplomatiques (car il n'est rien de tel à l'époque de Louis XII, ce qu'a encore rappelé Marion Pouspin dans Publier la nouvelle. Les pièces gothiques, histoire d'un nouveau média (XV'e-XVI ${ }^{e}$ siècles), Paris, Éditions de la Sorbonne, 2016, http://books.openedition.org/psorbonne/27111, chap. 6, § 22), mais comme le soutien spontané et appuyé du poète à la politique royale.

${ }^{7}$ Ils sont les dédicataires respectifs des Abus du Monde (Paris, Pierre le Dru, 1509) et de La Chasse du cerf des cerfz (s.l.n.d [Paris, october 1511], tous deux édités dans Euvres polémiques rédigées sous le règne de Louis XII, op. cit., p. 207-235.

${ }^{8}$ Cela suppose qu'aucun imprimeur peu scrupuleux ne pirate ses œuvres, d'où la nécessité d'obtenir un privilège royal pour les protéger : voir sur ce point Cynthia Brown dans Poets, Patrons and Printers. Crisis of Authority in Late Medieval France, Ithaca \& London, Cornell U. P., 1995. Elle montre que suite à l'impression abusive de son Chasteau d'Amours par Le Noir (ce dernier réécrit l'acrostiche final portant la signature de Gringore afin de s'attribuer le mérite de l'œuvre et de sa morale), Gringore s'efforce d'obtenir un privilège d'auteur pour pratiquement chacune de ses œuvres. Sans doute ce privilège se comprend-il également dans le cadre de la quête de reconnaissance menée par Gringore : solliciter un privilège d'auteur (et non d'éditeur, comme cela était alors plus usuel) est une façon de s'adresser aux élites en charge de l'octroyer et fonctionne aussi comme une marque d'autorité vis-à-vis du texte publié.

${ }^{9}$ Ces parutions ne sont alors pas contrôlées par la couronne selon Jean-Pierre Seguin (L'Information en France, de Louis XII à Henri II, Genève, Droz, 1961, p. 49) puis Marion Pouspin (Publier la nouvelle, op. cit., chap. 13 : «Les pièces gothiques d'actualité politique et militaire : des médias communicationnels, ou du faire-savoir au faire-être », p. 427-467). Même lorsque la plaquette est assortie d'un privilège, celui-ci est de très courte durée et vise à protéger le libraire (parfois vainement d'ailleurs) plus que l'auteur (souvent anonyme). Du reste, ce privilège ne signifie pas non plus que le pouvoir surveille particulièrement le contenu de ces imprimés. En 1507 et en 1509, cependant, Louis XII charge l'imprimeur lyonnais Noël Abraham de donner des nouvelles de ses expéditions génoises et vénitiennes.

${ }^{10} \mathrm{C}$ 'est ce que montrent Jennifer Britnell (Le Roi très chrétien contre le pape. Écrits antipapaux en français sous le règne de Louis XII, Paris, Classiques Garnier, 2011) et Nicole Hochner (Louis XII. Les dérèglements de l'image royale (1498-1515), Champ Vallon, 2013) : selon elles, ces écrits de propagande visent avant tout à flatter les oreilles du roi et à manifester le nombre de ses soutiens.

${ }^{11}$ Le traictie Intitule de la difference des schismes et des concilles de leglise. Et de la preeminence et vtilite des concilles de la saincte eglise Gallicane, Lyon, Etienne Baland pour Jean Richier, [mai 1511].

${ }^{12}$ Il n'a jamais été rémunéré par le pouvoir pour ses nombreuses œuvres de propagande sous les règnes de Louis XII et de François I I ${ }^{\text {er }}$, sinon pour les mystères qu'il a organisés et fait jouer à l'occasion d'entrées royales. Voir l'introduction de Cynthia Brown dans son édition de Pierre Gringore, Entrées royales à Paris de Marie
} 
Lemaire, en 1512, était déjà fort de sa position d'historiographe à la cour de Bourgogne, et avait, semble-t-il, déjà séduit Anne de Bretagne avec les vers des Épîtres de l'Amant vert. Gringore, bien qu'il semble avoir saisi l'importance de l'impact de la diffusion imprimée des écrits soutenant la politique étrangère de Louis XII bien avant Lemaire, est alors loin de jouir d'une telle autorité. Aussi s'efforce-t-il, comme nous allons le voir, de la construire dès sa première œuvre de propagande, en s'associant, sur le papier, à la couronne royale. Gringore choisit donc, pour les Lettres nouvelles de Milan, un support offrant rémunération, permettant de toucher une audience large, afin d'informer, mais aussi de célébrer la couronne, de manifester ses talents de poète et sa légitimité de propagandiste, de façon à suggérer, même indirectement, à un puissant lecteur (à la cour royale ?) de le prendre sous sa protection. Tout cela passe par un ajout significatif de vers aux informations en prose qui circulent déjà ou circuleront par ailleurs, dans un bulletin d'information savamment élaboré.

\section{Un support de promotion et d'auto-promotion}

Les Lettres nouvelles de Milan relèvent de la production de bulletins d'information. Comme l'expliquent Jean-Pierre Seguin et Marion Pouspin dans leurs études sur la diffusion de ces bulletins à l'orée $\mathrm{du} \mathrm{XVI}^{\mathrm{e}}$ siècle $^{13}$, cette production imprimée se développe à la fin du siècle précédent et aborde en particulier des événements importants des guerres d'Italie ou de la vie politique intérieure française. Les bulletins d'information consistent souvent en des lettres, parfois accompagnées de vers. L'éditeur Le Caron était alors spécialisé dans ce type de production et se partageait l'essentiel du marché des livret gothiques avec Trepperel. C'est certainement la raison pour laquelle Gringore ne se tourne pas, pour cette publication, vers Philippe Pigouchet - célèbre éditeur d'Heures et d'œuvres morales - alors qu'il avait collaboré un an plus tôt avec lui pour éditer sa première œuvre, le Chasteau de Labour, et que cette collaboration se renforcera à la fin de l'année 1500 avec la parution d'une nouvelle œuvre morale de Gringore : le Chasteau d'Amours ${ }^{14}$.

Sur la page de titre des Lettres nouvelles de Milan, les trois lettres en prose sont annoncées en grands caractères (les « regretz du seigneur Ludovic » figurent en plus petits caractères). Même si elles ont peut-être été adaptées par Gringore ou son éditeur ${ }^{15}$, elles sont vraisemblablement authentiques : leur provenance royale constitue un puissant argument de véridicité. Le reste, occupé par les vers de Gringore, apparait comme un ajout voire un ornement apposé à la transcription épistolaire, qui contient l'essentiel des informations. Ce dispositif, explique Jean-Pierre Seguin, n'a rien de particulièrement original ; les recensions de Marion Pouspin permettent même d'établir que ces deux formes de communication - lettre et poème de circonstance - seront les plus employées dans les brochures gothiques d'information durant les décennies $1500-1510^{16}$. L'éditeur s'est sans doute emparé de lettres du roi et de son secrétaire informant des victoires françaises et de la capture de l'ennemi milanais ; Gringore a ensuite ajouté ses propres vers à ce projet éditorial, de façon à attacher

d'Angleterre (1514) et Claude de France (1517), Genève, Droz, 2005, p. 11-13 : associé au charpentier Jean Marchant, Gringore composa des mystères, ou tableaux vivants, pour les entrées de l'archiduc Philippe d'Autriche en 1501, du légat papal Georges d'Amboise en 1502 et d'Anne de Bretagne en 1504. Les deux hommes collaborèrent également pour l'ensemble de l'entrée de Marie d'Angleterre et celle de Claude de France.

${ }^{13}$ L'Information en France, op. cit. (voir en particulier p. 50-51 sur Le Caron) et Publier la nouvelle, op. cit (voir notamment chap. 6 : «L'émergence d'une presse d'actualité », p. 159-196).

${ }^{14}$ Sur les traces de ces collaborations dans les œuvres imprimées de Gringore, voir Cynthia Brown, Poets, Patrons and Printers, op. cit., p. 79-83.

${ }^{15}$ Voir l'édition de Cynthia Brown (op. cit., p. 63) ainsi que Jean-Pierre Seguin (op. cit., p. 26), selon lequel les éditeurs pouvaient remanier ces informations. René de Maulde La Clavière (op. cit., p. 267) puis Enea Balmas (op. cit., p. 51-57) détaillent par ailleurs les incohérences dans la datation de ces lettres.

${ }^{16}$ Publier la nouvelle, op. cit., tabl. 8. 
son nom à celui de la couronne à travers cet imprimé ${ }^{17}$. Les vers qui suivent ces lettres ont une portée encomiastique : l'éloge justifié succède à l'information. Plus exactement, dans la mesure où la troisième lettre, non signée, s'attache déjà à la célébration de la victoire française, à Paris, où se trouvait Gringore, on peut avancer qu'il est déjà l'auteur de ce texte : cette troisième brève lettre fonctionnerait alors comme une transition entre la prose neutre et factuellement véridique, dépourvue de commentaires de l' « acteur », narrant et résumant la célébration, et les vers qui déploient justement ce triomphe.

Dans ce dispositif relativement conventionnel, deux éléments confèrent toutefois une signification accrue à la présence de ces vers à la gloire du roi et de son entreprise militaire. D'une part, dans la plupart des bulletins d'information, les vers ne sont pas signés. Pourtant, Gringore prend soin d'insérer son nom dans la dernière strophe, sous forme d'acrostiche :

\author{
Gentilz Françoys, soyez de la victoire \\ Remercïans Jesus le Createur. \\ Il nous appert que l'euvre meritoire \\ Nous vient du Ciel. Dieu est nostre adjusteur. \\ Gloire, triumphe, magnificence, honneur \\ Ont conquesté a Milan [les] gens d'armes. \\ Regretz, souspirs Ludovic en son cueur \\ En a souvent, et pleure maintes larmes. (v. 319-326)
}

Une telle mise en exergue de l'auctorialité du bulletin suggère que le contenu proprement informatif n'est pas l'essentiel du texte ${ }^{18}$. Sa valeur réside dans la figure de l'encomiaste qui émerge à côté de la transcription des lettres du roi et de son secrétaire Robertet.

Cette valorisation de l'autorité du poète est d'autre part renforcée par le contexte éditorial de la parution des Lettres nouvelles de Milan. En effet, le texte signé par Gringore fait pendant à une publication très proche par son titre et son contenu : Les Lettres nouvelles de Milan envoyées au Roy nostre sire de par Monseigneur de La Trimouille, touchant la prise de Ludovic, avec l'amende honorable faicte par les Milannoys au Roy... à la personne de Monseigneur le cardinal d'Amboyse, lieutenant général du Roy... au pays du Milannoys. Cette plaquette, réunissant des épîtres rédigées par la Trémoille et adressées au roi, narre également la progression de l'armée française en Milanais, la prise de la ville ainsi que la capture du duc, de façon bien plus détaillée que dans le livret portant le nom de Gringore ${ }^{19}$. Figurent en outre les requêtes adressées par les habitants et les grâces accordées par Louis XII, à travers un nouvel échange d'épîtres cette fois avec le cardinal d'Amboise. Bien que publiée sans lieu ni date, cette plaquette semble avoir été éditée par Le Caron également : les pages de titres présentent des similarités frappantes dans leur typographie et par la gravure utilisée (qui est exactement la même, représentant un homme captif conduit hors d'une ville). Aussi Jean-Pierre Seguin l'attribue-t-il sans hésiter à Le Caron ${ }^{20}$. Le fait qu'un éditeur republie un bulletin d'information sur un même événement, pourvu que le contenu soit enrichi, n'est pas une pratique inédite ${ }^{21}$. Par le sujet qu'elles traitent, les deux publications sont assurément très proches, sans que l'on puisse déterminer leur ordre de parution. Quel

\footnotetext{
${ }^{17}$ Nous n'avons nulle trace d'une circulation manuscrite ou imprimée séparée et indépendante de ces textes, ce qui étaye l'hypothèse d'un projet éditorial mené par Le Caron, où il mettrait en valeur un document émanant de la couronne au moyen de vers composés par un collaborateur poète.

${ }^{18}$ Sur les valeurs de l'acrostiche durant cette période, voir Nathalie Dauvois, « Acrostiches et anagrammes du nom d'auteur à la Renaissance. Évolution et significations », Quant l'ung amy pour l'autre veille. Mélanges offerts à Claude Thiry, éd. T. Van Hemelryck et M. Colombo Timelli, Turnhout, Brepols, 2008, p. 92-99.

${ }^{19}$ Selon Jean D'Auton, c'est par ces lettres que le roi fut informé de la capture de Ludovic (Chroniques de Louis XII, op. cit., p. 266).

${ }^{20}$ L'Information en France, op. cit., p. 59-60. Dans cette autre plaquette, on ne retrouve toutefois pas les lettrines figurant dans les publications clairement attribuées à Le Caron.

${ }^{21}$ Ibid., p. 25-28.
} 
qu'il soit, ces deux plaquettes d'information entretiennent moins des rapports de concurrence que de complémentarité : aux détails des informations données dans les lettres de La Trémoille, répondent l'autorité d'une épître signée de la main du roi ainsi qu'une transposition éthique et poétique triomphale du contenu factuel des lettres officielles par Gringore. L'espace des vers se distingue donc particulièrement et revêt une fonction autopromotionnelle, mais c'est aussi par là que l'information se fait célébration.

\section{L'amplification encomiastique}

Afin de conférer une rhétorique démonstrative à l'information ainsi réitérée, les vers fonctionnent comme une amplification des lettres qui précèdent immédiatement. Tout d'abord, dans la partie versifiée intitulée «S'ensuyt le debat des François contre le sire Ludovic. Avec les regretz d'iceluy et complainte des Milannoys », des informations sont précisées, à propos de la capture de Sforza (on apprend qu'il était déguisé en moine), ou bien à propos des remords de Milan, alors que la ville admet ses vices (cupidité et lâcheté) et reconnaît Louis XII comme son vrai roi :

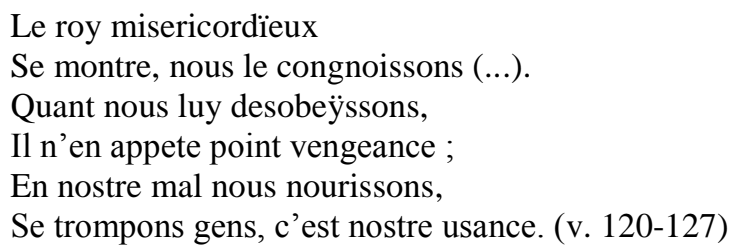

Ces éléments sont tous à la charge des Milanais et de leur ancien duc. Les vers composés par Gringore sont par ailleurs proches des lettres qui furent publiées dans la deuxième plaquette, Les Lettres nouvelles de Milan envoyées au Roy nostre sire de par Monseigneur de La Trimouille... : qu'elles furent imprimées ou non au moment de la rédaction de ses vers, Gringore avait sans doute accès à ces informations dans le cercle de l'éditeur Le Caron. Ainsi, dans la mise en scène où dialoguent Italiens repentis et Français victorieux mais miséricordieux de Gringore, se retrouvent «l'amende honorable faicte par les Milannoys » ainsi que la réponse du cardinal d'Amboise, qui évoque longuement la clémence de Louis XII.

Un autre aspect de l'amplification consiste à appliquer aux événements une signification morale et eschatologique. Dans cet exemple, la victoire française devient celle d'un champion de la chrétienté contre un usurpateur :

Dieu a aydé au trescrestien roy

Quant son arroy a si tresbien conduit

Car selon droit, raison, justice et loi

Ludovic voy happé en desarroy (...). (v. 17-20)

La dimension chrétienne de la victoire est un lieu commun des poètes de cour pour faire l'éloge de leur prince : il apparaît par exemple dans les comptes rendus de campagnes militaires d'André de la Vigne ou Jean Marot $^{22}$.

En précisant la portée morale des événements et en faisant l'éloge du roi, l'amplification revêt enfin un troisième aspect puisque la grandeur du monarque se reflète dans la virtuosité

\footnotetext{
${ }^{22}$ Voir par exemple Le Voyage de Gênes de Jean Marot, écrit en 1507. Quand les Génois vaincus remercient Louis XII pour sa pitié, ils attribuent aussi sa victoire à la volonté divine : «Et que du bien j'ay plus que ne merite / Quant le vray Dieu de sa grace me herite / D'ung si hault prince comme le roy de France » (éd. Giovanna Trisolini, Genève, Droz, 1974, v. 1217-1219).
} 
des vers eux-mêmes ${ }^{23}$. Ainsi, les premières strophes mobilisent toutes sortes de rimes extraordinaires, qui rehaussent le propos et manifestent le talent technique du poète. Par exemple, les vers 17-20 déjà mentionnés présentent des rimes batelées, brisées et même des vers léonins (nous soulignons) :

Dieu a aydé au trescrestïen roy

Quant son arroy a si tresbien conduit

Car selon droit, raison, justice et loi

Ludovic voy happé en desarroy (...). (v. 17-20)

Ailleurs, Gringore emploie la rime équivoque :

Nul ne peut entendre voz cas,

Quant il auroit l'opinïon

De quatre cens mille advocas. (v. 101-103)

Plus loin, mais toujours dans le débat des Français et des Italiens, l'emploi de la figure dérivative confine à la rime annexée :

Mais ilz [les escus] seront tous desamassez [sic],

Et les amassans en souffrance,

Les Françoys ont bonne esperance (...). (v. 114-116)

Les exemples sont nombreux dans cette partie du texte. Les vers du poète ne servent pourtant pas seulement à une démonstration de première et de seconde rhétorique, d'emploi habile des lieux de l'éloge et de virtuosité technique. C'est parce qu'ils ne sont pas confinés à la vérité factuelle ou à l'hic et nunc, comme l'étaient les lettres, qu'ils peuvent porter une plus haute signification en se situant sur le plan des valeurs. L'éloge éclatant du roi et des Français, dont les vers ornés sont mimétiques, élève la célébration du fait militaire aux plans éthique et esthétique. En tant que poète, Gringore est d'autant plus capable d'édifier une audience en quête de nouvelles fraiches.

\section{Propagande ou flatterie?}

La prose n'est pas pour autant complètement dévaluée : sa forme épistolaire la situe dans une vérité factuelle ${ }^{24}$ reposant sur des témoignages de première main. D'ailleurs, à l'inverse

\footnotetext{
${ }^{23}$ Sur l'adéquation entre l'éclat du registre encomiastique et celui de la versification, voir François Cornilliat, "Or ne mens ». Couleurs de l'éloge et du blâme chez les « Grands Rhétoriqueurs », Paris, Champion, 1994.

${ }^{24}$ Dans le Voyage de Naples, La Vigne semble ainsi opposer les passages en vers, épidictiques, décrivant les entrées triomphales de Charles VIII aux passages en prose motivés par la recherche de la vérité, qu'il lie au critère rhétorique de la brièveté (qui suppose de raconter les faits clairement, dans l'ordre et sans détour), ce que les exigences métriques du vers ne permettaient pas : « Pource qu'en la matiere presente y a plusieurs choses qui bonnement ne se pourroient acoustrer en ryme si briefvement comme l'en pourroit faire en prose, a raison de ce que la matiere est de grant efficace et que plusieurs choses y sont comprinses qui requierent estre escriptes selon qu'elles ont été dictes, proferees ou venues, allees et executees, aussi pour les noms des personnaiges, des lieux, du temps et des termes tenus en ceste affaire ; parquoy au plus brief que je pourray, selon la verité, en ensuivant l'abrégé de ma ryme, j'en diray en prose ce que je verrai qui sera bon de dire, sans plus. » (Le Voyage de Naples, éd. Anna Slerca, Milan, Vita e Pensiero, 1981, p. 281 ; voir aussi les vers 4531-4544, p. 251, qui donnaient déjà ces arguments en vers). Les vers, comme les jugements élogieux, s'apparentent donc à un surcroît épidictique par rapport à la prose purement et plus proprement véridique. Sur le Voyage de Naples et la présence discutée des vers, par rapport à La Ressource de la chrestienté allégorique dont il est le pendant historique, voir Cynthia Brown, The Shaping of History..., op. cit., et "Mémoire et histoire : la déformation de la réalité chez les Rhétoriqueurs à la fin du Moyen Âge », dans Jeux de mémoire. Aspects de la Mnémotechnie médiévale, éd. B. Roy et P. Zumthor, Paris/Montréal, Vrin/Les Presses de l'université de Montréal, 1985, p. 43-53.
} 
des auteurs des lettres en prose dans son imprimé (et à l'inverse de La Trémoille et du cardinal d'Amboise, qui ont rédigé les lettres sur Milan imprimées au même moment dans la seconde plaquette), Gringore n'a pas été témoin des faits qu'il transcrit et interprète dans ses vers. Par conséquent, d'où tire-t-il sa légitimité à expliquer et célébrer la situation à Milan ? En quoi est-il différent d'un simple flatteur, s'il n'est pas présent auprès du roi pour attester les valeurs qu'il soutient ${ }^{25}$ ? Dans les Lettres nouvelles de Milan, Gringore offre dans ses vers ses propres réponses à ce problème de la flatterie par ailleurs récurrent dans les travaux des Rhétoriqueurs, à travers un nouveau montage significatif, cette fois entre le débat des Français et des Italiens, et le discours de Ludovic Sforza, qui se présente de prime abord sous forme de complainte. De toute évidence, et par contraste avec les authentiques lettres en prose, ce discours a été complètement forgé par Gringore : comment peut-il refléter une quelconque vérité ?

Ludovic Sforza, s'exprimant simplement et pathétiquement dans des vers à présent dépourvus des ornements saillants relevés chez les Français et les Italiens, rappelle certains éléments de la victoire française, reconnaît ses propres défauts et fait l'éloge de celui qui l'a vaincu :

J'ay entreprins par subtile malice De mettre hors les Françoys d'Ytallie ;

Mais il est force que le roy me nourrice

Et que en sa grace humblement me humilie. (v. 166-169)

Jusque là, Ludovic ne semble que réitérer le contenu du débat qui s'est tenu entre Français et Italiens. Mais ensuite, il rejette ses fautes sur la Fortune et sur les Lombards eux-mêmes. Comme l'explique Cynthia Brown dans son édition, il ne cause alors plus la pitié, mais soulève l'indignation du lecteur envers un homme hypocrite qui va jusqu'à accuser son propre peuple :

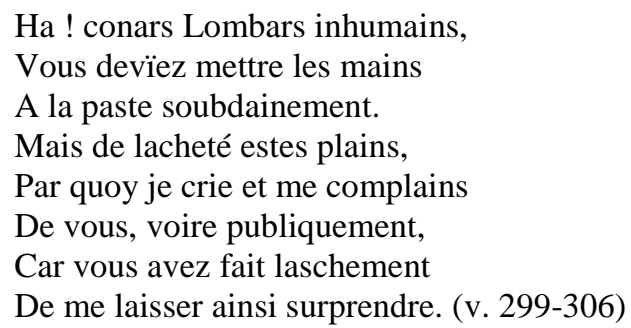

Alors que l'apparition de la deuxième personne signale la véhémence de la condamnation, le discours passe de la complainte à l'accusation, du registre démonstratif au judiciaire. Ludovic Sforza apparaît ainsi comme le double inversé de Louis XII : en effet, dans les vers 120-127 déjà cités, le roi de France pardonnait à ses sujets qui s'étaient soulevés et ne les blâmait pas outre mesure. Même s'il n'a pas encore le surnom de «père du peuple » en 1500, l'attention toute paternelle que Louis XII porte à ses sujets (du moins selon ses porte-paroles), en leur épargnant les pillages notamment, montre déjà sa magnanimité et suggère que son autorité est d'autant plus grande qu'il choisit de ne pas en faire de démonstration brutale ${ }^{26}$.

\footnotetext{
${ }^{25}$ C'est l'argument clé de Jean Marot, par exemple, quand il se défend d'enjoliver les victoires de Louis XII à Gênes et à Venise. Ainsi, même si Le Voyage de Gênes peut sembler trop flatteur pour étre véridique, la qualité de témoin de Marot vient garantir l'éloge : «Impossibl'est que j'en creusse le tiers, / Mais je y estoyes, parquoy je le dois croire » (op. cit., v. 585-586).

${ }^{26}$ Nicole Hochner développe ce point dans Louis XII. Les dérèglements de l'image royale (1498-1515), op. cit., notamment « Chapitre 5 - Prince de vertus et père du peuple », p. 176-215.
} 
Or la modification de l'éthos de Ludovic (de l'humilité à la colère) ainsi que de son motus (de la pitié à l'indignation), qui révèle sa vraie nature et porte en creux un éloge de Louis XII, a lieu de part et d'autre de deux rondeaux. Ils traitent tous deux de l'hybris du duc défait ainsi que de la victoire Française et ont respectivement pour rentrement : «Or suis je prins » (v. 249) et «Le More est prins » (v. 264). Ces rondeaux semblent se répéter et pourtant le récit des événements passe de la première à la troisième personne, ce qui aboutit à une nouvelle interprétation de l'attitude de Sforza: ce qui était «ignorance» (v. 255) ou impuissance (v. 258) dans le premier rondeau devient «ruse» (v. 265) dans le second. Le vice est dévoilé, d'une manière comparable au dévoilement de ses habits par Mère Sotte à la fin de la sottie du Jeu du Prince des Sotz et de Mère Sotte, alors qu'elle passait jusqu'à présent pour Mère Église. Dans les Lettres nouvelles de Milan, ce glissement de la première à la troisième personne dans les rondeaux, de même que, plus haut, le passage de la prose aux vers, représente la distance du poète par rapport aux événements qu'il commente. Or une telle distance donne de la perspective et garantit la vérité. Elle a permis à Gringore de montrer Ludovic sous son vrai jour hypocrite et colérique. Il est significatif que le rôle crucial d'un poète distant pour dire la vérité émerge dans les rondeaux, c'est-à-dire dans une forme poétique plus élaborée que les dizains qui les entourent. De ce point de vue éloigné et poétique, l'écrivain s'avère plus clairvoyant pour dresser le portrait des hommes et en tirer des conclusions sur le légitime usage du pouvoir - il se fait ainsi l'exact opposé du flatteur.

\section{Des choix stylistiques entre pédagogie et éloge}

À travers un bulletin d'information destiné à un public urbain, plus ou moins lettré mais avide de nouvelles d'Italie, Gringore se pose en potentiel encomiaste officiel de la couronne, talentueux et réfléchi ${ }^{27}$. Or ces deux objectifs promotionnels et auto-promotionnels supposent un traitement de l'elocutio parfois contradictoire, ce que révèlent les vers des Lettres nouvelles de Milan dans leur hétérogénéité formelle et stylistique. Cela apparaît dans la redondance des propos contenus entre le débat entre Français et Italiens d'une part et les regrets de Ludovic d'autre part : cette répétition de deux passages versifiés ne relève en effet pas seulement d'une variation permettant à Gringore de fonder son autorité d'encomiaste à distance, mais fait aussi apparaître une divergence stylistique significative. Le discours du More est peu orné et contraste avec le débat des Français et des Italiens. Il met en scène une personne réelle et non un collectif abstrait. Ses strophes présentent peu de rimes extraordinaires mais beaucoup d'enjambements :

Or esse par lache couraige

Que je suis tenu en servage,

Dont grandement je me soussie ;

C'est au prejudice et dommaige

Des Milannoys. Vent ou oraige

Ont gasté ma nigromancie (...). (v. 289-294)

L'écriture de Gringore se fait plus prosaïque pour être mimétique d'un vrai discours - sans revenir à la prose neutre et informative des lettres. Mais cette inflexion stylistique et technique s'explique sans doute également par souci de clarté, dans le cadre d'une oralisation du texte, à la cour mais surtout dans les villes. Selon Jean-Pierre Seguin puis Marion Pouspin ${ }^{28}$, en effet, les bulletins d'information étaient souvent lus à ceux qui ne savaient pas lire (voisins ou

\footnotetext{
${ }^{27}$ Nicole Hochner en a souligné la solidité dans son article «Pierre Gringore : une satire à la solde du pouvoir ?», Fifteenth-Century Studies, 2001, vol. 26, p. 102-120, ainsi que dans son ouvrage Louis XII. Les dérèglements de l'image royale (1498-1515), op. cit.

${ }^{28}$ L'Information en France, op. cit, p. 51-52 et Publier la nouvelle, op. cit., introduction, $\S 12$.
} 
autres proches de l'acheteur), voire criés. Mais les échos rimiques des rimes batelées, brisées ou des vers léonins perturbent la perception orale du rythme du vers ; tandis que les rimes équivoquées font entendre des termes semblables alors que leur intérêt réside précisément dans la dissimilarité visuelle ${ }^{29}$. Dans cette perspective, le débat entre Français et Italiens ne convient guère à cette oralisation pour les lecteurs non lettrés des villes. La plainte et l'accusation du More répètent certes l'information et sa charge morale, mais le discours du duc vaincu rend les deux plus explicites, car énoncés dans un style plus simple. En conséquence, il y a un contraste, dans les Lettres nouvelles de Milan, entre un style orné adapté à la glorification du roi et à l'attention de lecteurs coutumiers de ces démonstrations de virtuosité - ceux-là mêmes qui pourraient contribuer à la carrière du jeune Gringore -, et un autre plus prosaïque qui peut sembler plus évident et plus sincère, plus à même d'édifier son audience par sa clarté, ou du moins atteindre ceux qui n'auraient pas assez de culture littéraire pour saisir les subtilités de la haute poésie épidictique - ceux qui assurent une diffusion massive du texte. Deux techniques poétiques correspondent donc à deux stratégies et à deux catégories de public.

Cette distinction et les difficultés qu'elle impose au poète seront explicitées à propos des complexes tableaux allégoriques que Gringore compose lors des entrées royales. Ils sont spécifiquement adressés aux membres du cortège royal et Gringore se charge d'en exposer la richesse symbolique dans de précieux manuscrits offerts aux reines dont il a préparé l'entrée : Marie d'Angleterre en 1514 et Claude de France en 1517. Alors que des comptes rendus publiés circulaient, Gringore explique qu'il rédige lui-même le récit de l'entrée et la description des tableaux car, selon lui, le public (et notamment ceux qui ont composé les comptes rendus imprimés) ne les entendait guère :

\footnotetext{
Rememorant que aucuns escripts ont faictz

De ton entree, ignorans les effectz,

Ditz et raisons contenuz en icelle,

Que soubz telz gens la verité se celle,

Cuidant savoir la pensee de l'acteur,

Qui de ce cas a esté inventeur,

Et ont voulu si noble entree extraire,

Non pas au vray, mais du tout au contraire,

Par quoy je en vueil faire narration

Selon ledit interpretation

De je qui suis nommé Pierre Gringoire,

Comme verras par ditz et vraye histoire ${ }^{30}$.
}

Outre le désir de mettre en valeur son nom et son œuvre, Gringore manifeste ici sa conscience que trop de complexité, technique ou symbolique, n'est pas entendue par le grand public ${ }^{31}$.

D'autres œuvres de Gringore, postérieures aux Lettres nouvelles de Milan, corroborent l'idée d'une répartition des styles selon les milieux visés. Ils ne cohabitent guère plus au sein d'une seule œuvre. Alors que La Piteuse Complainte de la Terre Sainte de l'été 1500 offre, comme dans le débat des Italiens et des Français des Lettres nouvelles de Milan, des rimes batelées, couronnées, léonines, ou encore des jeux sur la dérivation ou la paronomase, L'Entreprise de Venise de 1509 en est globalement dépourvue : les strophes sont en outre régulières et les enjambements fréquents, rappelant plutôt la partie du discours de Ludovic Sforza des Lettres nouvelles de Milan. Il en va de même pour L'Union des Princes du printemps 1509, pour L'Espoir de Paix de février 1511 ou L'Obstination des Suysses, paru entre juin 1512 et juin 1513. Cynthia Brown suggère que Gringore, précipité par les

\footnotetext{
${ }^{29}$ Voir encore les démonstrations de François Cornilliat dans Or ne mens, op. cit.

${ }^{30}$ Entrées royales à Paris de Marie d'Angleterre (1514) et Claude de France (1517), op. cit., p. 157, v. 1-12.

${ }^{31}$ Voir aussi l'introduction de Cynthia Brown, ibid., p. 58-85.
} 
événements, a manqué de temps lorsqu'il composa ces pamphlets: une écriture rapide expliquerait une telle simplicité ${ }^{32}$. Mais on peut aussi avancer que le poète y met de côté la recherche de la virtuosité pour promouvoir sincérité et clarté, optant pour une fin informative plutôt qu'encomiastique, sans doute plus destinée à un large public qu'à une élite. Ce n'est pas tant là une évolution de Gringore, délaissant peu à peu les ornements de la seconde rhétorique, qu'un choix conscient adopté en fonction du public visé. Ainsi, le pamphlet intitulé La Chasse du cerf des cerfs, accablant le pape Jules II, que Gringore publia en 1511, fait à nouveau apparaître jeux de mots (l'ensemble de la composition repose sur un calembour entre «cerf» et «serf», terme que saint Grégoire employait pour qualifier la fonction papale $^{33}$ ) et virtuosité métrique. Or la diffusion de ce texte est d'abord limitée à son dédicataire, choisi et lettré : l'évêque de Cahors. Dès que Gringore cible un lecteur éminent pour solliciter une rétribution ponctuelle ou une protection définitive - l'elocutio se fait à nouveau virtuose, de façon à créer une complicité avec un lectorat choisi, et à constituer une autorité d'écrivain qui repose moins sur une interprétation pédagogique des faits (inutile) que sur une maîtrise technique et une charge démonstrative. De ce point de vue, la réitération des nouvelles de Milan avec différents traitements du vers marque une tension entre des pratiques poétiques de propagande adaptées à des publics différents et finalement peu compatibles.

Pour conclure, la première œuvre de propagande de Gringore se présente comme une somme: les variations formelles et énonciatives autour du récit d'un même événement manifestent des stratégies rhétoriques différentes, entre information et célébration, destinées à toucher des publics non moins hétérogènes, des plus modestes habitants des villes aux plus éminents princes et prélats, protecteurs potentiels. Si un tel assemblage entre prose et vers illustre les diverses facettes de la légitimité de Gringore à se faire encomiaste officiel, il révèle aussi, par les tensions au sein du traitement des vers, les choix qu'impose une diffusion nouvelle et non encadrée des textes à la gloire du monarque.

Ellen Delvallée CNRS (UMR 5316 Litt\&Arts, Université Grenoble Alpes)

\footnotetext{
32 Euvres polémiques, op. cit., p. 73-74. En effet, certains vers sont fautifs voire manquants, mais la précipitation est peut-être surtout celle de l'imprimeur.

33 Saint Grégoire (d'ailleurs cité en exemple dans le texte) emploie l'expression «servus servorum Dei», empruntée à saint Augustin : voir les notes de Cynthia Brown, dans Euvres polémiques, op. cit., p. 230. On pourrait aussi évoquer en exemple d'autres jeux sur les mots, notamment dans ces vers par ailleurs étudiés par François Cornilliat (Or ne mens, op. cit., p. 316-318): «Veneurs n'ont point cette leçon aprise / Que l'on corne du serf des cerfz la prise, / Sans qu'il soit prins. Quelq'un voulut corne[r] / La mort du serf; les autres par faintise / Cornerent lors en oyant sa devise. / Lors voulurent tous leurs cors encorner. / Plusieurs cuydoient en cornant escorner, / Mais on congneut la cornerie en fin : / Affiné est aucuneffois le fin. » (CEuvres polémiques, op. cit., p. 224, v. 154-162).
} 